\title{
Effect of feedback carrier excitation on LED external quantum efficiency
}

\author{
L. Kučera, C.Sc., J. Macháč, and J. Mišek, C.Sc.
}

Indexing terms: $\quad$ Semiconductor devices and materials, Diodes, Light-emitting diodes

\begin{abstract}
Self-absorption of photons generated by prior luminescence processes affects both the steady-state and transient characteristics of LEDs. In this study we confine ourselves to one of the most important steadystate parameters - external quantum efficiency. It is shown that the photon self-absorption increases the injected carrier concentration, and consequently the emitted photon flux. This results in an increase in LED external quantum efficiency. A detailed analysis of this phenomenon is presented, and a simplified evaluation of its influence on the external quantum efficiency is given. Our calculation also takes into account multiple internal reflection of light generated inside the LED. The obtained results are applied to a diffused GaAs ( $\mathrm{Zn}, \mathrm{Te}$ ) surface-emitting LED. It is estimated that the feedback carrier excitation (re-excitation) due to selfabsorption increases the LED external quantum efficiency by about $60 \%$ and multiple internal reflections by about $12 \%$.
\end{abstract}

\section{Introduction}

In an LED with a direct bandgap structure, a large number of photons generated by luminescence processes are subsequently reabsorbed within the LED's volume [1]. This photon absorption results in an excitation of carriers from the valence band to the conduction band. Excited carriers can again recombine radiatively. This feedback carrier excitation (reexcitation) causes a change in output photon flux, and consequently affects both the steady-state and transient characteristics of the LED. In our study we confine ourselves to the steady-state external quantum efficiency of the LED.

Re-excitation has recently been described by Kameda and Carr [2], Stern and Woodall [3], Kuriyama et al. [4], Epifanov et al. [5] and Kučera et al. [6]. Kuriyama et al. analysed the contribution of the re-excitation to the external quantum efficiency of $\mathrm{GaAs}-\mathrm{Al}_{x} \mathrm{Ga}_{1-x}$ As heterostructures [4]. The influence of re-excitation on diffusion lengths of minority carriers and the minority-carrier concentration distribution in GaAs epitaxial layers excited by intensive light beam has been studied by Epifanov et al. [5]. The influence on emission spectrum shape has also been studied [6] .

In our paper a theoretical model of re-excitation is studied. The main purpose has been to describe the influence of the re-excitation on the LED external quantum efficiency. The presented theory also includes multiple reflections of light generated in the diode, as this effect cannot be neglected in many LED structures.

\section{Theory of re-excitation}

In any analysis of re-excitation we need to determine the photon generation rate $g(x, \epsilon)$, where $x$ is the position and $\epsilon$ is the photon energy. This function represents the number of photon generated per unit volume per unit time, and may be evaluated by a self-consistent method. In the first approximation we write the generation rate as $g^{(1)}(x, \epsilon)$, and do not consider re-excitation. This function $g^{(1)}$ is then used to determine the photon absorption rate $v(x, \epsilon)$ giving the number of photons absorbed per unit volume per unit time. This value of photon absorption rate is then used to find the second approximation to the generation rate $g^{(2)}(x, \epsilon)$ from a modified continuity equation, and taking into account the first approximation of the photon absorption rate. The procedure may be repeated to find a new photon absorption rate and a third approximation to the generation rate. It may

Paper 1737I, first received 2 nd July and in final form 30 th October 1981

The authors are with the Institute of Radio Engineering \& Electronics, Czechoslovak Academy of Sciences, Lumumbova 1, 18251 Prague 8, Czechoslovakia be shown that the third approximation does not contribute to the generation rate significantly. For practical purposes then, it is sufficient to confine this procedure to the first two steps and to consider the function $g^{(2)}$ as the resultant function, the superscript (2) denoting the second approximation only.

The calculation is made under the simplifying assumptions that the junction under consideration is abrupt, and that dopants are fully ionised and uniformly distributed. Analysis is performed for the case of low-level injection, with uniform distribution of the current density across the junction area. The generation region thickness is much less than transverse dimensions. In this case the problem may be solved using a one-dimensional model of the diode (Fig. 1).

The first approximation of the photon generation rate without re-excitation, is a solution of a modified continuity equation and may be written as [8]

$$
\begin{aligned}
& g^{(1)}(x, \epsilon)=f(\epsilon) g_{0}(x)=A f(\epsilon) e^{-x / L_{n}} \\
& A=\frac{\eta S I}{e L_{n}} \frac{1}{1+\frac{\tau_{n} N_{A} L_{p}}{\tau_{p} N_{D} L_{n}}}
\end{aligned}
$$

where $f(\epsilon)$ is a generation spectrum normalised by the relation

$$
\int_{0}^{\infty} f(\epsilon) d \epsilon=1
$$

$\tau_{n}, \tau_{p}, L_{n}, L_{p}$ are lifetimes and diffusion lengths of minority electrons and holes in the $p$ - and $n$-regions of the diode, respectively, $N_{A}$ and $N_{D}$ are acceptor and donor concentrations, respectively, $e$ is the electron charge, $\eta$ the internal quantum efficiency, $S$ the $p-n$ junction area, $I$ the driving current, and $\epsilon$ is the photon energy.

The second approximation of the photon generation rate, taking re-excitation into account, may be obtained from a modified continuity equation

$$
\frac{d^{2} g^{(2)}(x, \epsilon)}{d x^{2}}-\frac{g^{(2)}(x, \epsilon)}{L_{n}^{2}}=-\frac{\eta}{L_{n}^{2}} r_{e}(x)
$$

with boundary conditions at $x=0$ and $x=1_{p}$

$$
\begin{aligned}
g^{(2)}(0, \epsilon) & =g^{(1)}(0, \epsilon)+\eta r_{e}(0) \\
g^{(2)}\left(l_{p}, \epsilon\right) & =g^{(1)}\left(l_{p}, \epsilon\right)+\eta r_{e}\left(l_{p}\right)
\end{aligned}
$$

where $l_{p}$ is the $p$-region thickness and $r_{e}(x)$ the carrier excitation rate having the form

$$
r_{e}(x)=\int_{0}^{\infty} h(\epsilon) v(x, \epsilon) \bar{d} \epsilon
$$


where $v(x, \epsilon)$ is the photon absorption rate determining the number of absorbed photons per unit volume per second, $h(\epsilon)$ is the quantum efficiency of an internal photoelectric phenomenon, e.g. the number of excited free carriers per absorbed photon. A variable $h(\epsilon)$ can be represented for direct bandgap semiconductors by a function proportional to an absorption coefficient of the generation region $\alpha_{p}$

$$
h(\epsilon)=k \alpha_{p}(\epsilon)
$$

where the constant $k$ may be determined from the normalisation condition

$$
k \alpha_{p}\left(\epsilon>\epsilon_{G}\right)=1
$$

$\epsilon_{G}$ is the bandgap energy.

The photon absorption rate can be divided into two parts

$$
v(x, \epsilon)=v_{i}(x, \epsilon)+v_{r}(x, \epsilon)
$$

where $v_{i}(x, \epsilon)$ is the part determining absorption of photons prior to reflection and $v_{r}(x, \epsilon)$ is the photon absorption rate of reflected photon flux. These two parts may be written in the form [6]

$$
v_{i, r}(x, \epsilon)=-\int_{-\infty}^{\infty} \int_{-\infty}^{\infty} \int_{0}^{l_{p}}\left(\operatorname{div}_{x, y, z} G_{i, r}\right)_{\begin{array}{r}
y=0 \\
z=0
\end{array}} d \xi_{x} d \xi_{y} d \xi_{z}
$$

where $\xi_{x}, \xi_{y}$ and $\xi_{z}$ are auxiliary co-ordinates (Fig. 1).

$G_{i}$ and $G_{r}$ are photon flux densities at the point $(x, 0,0)$, contributed by the volume element $d V=d \xi_{x} d \xi_{y} d \dot{\xi}_{z}$, with co-ordinates $\left(\xi_{x}, \xi_{y}, \xi_{z}\right)$ :

$$
\begin{aligned}
& G_{i}\left(x, \xi_{x}, \xi_{y}, \xi_{z}, \epsilon\right)=g^{(1)}(x, \epsilon) \frac{1}{4 \pi r^{2}} e^{-\alpha_{p}(\epsilon) r} \frac{r}{|r|} \\
& G_{r}\left(x, \xi_{x}, \xi_{y}, \xi_{z}, \epsilon\right)=g^{(1)}(x, \epsilon) \frac{\bar{R}}{4 \pi\left(r_{1}+r_{2}\right)^{2}} \times \\
& e^{-\alpha_{p}(\epsilon)\left(r_{1}+r_{2}\right)} \frac{r_{2}}{\left|r_{2}\right|}
\end{aligned}
$$

the values of lengths $r, r_{1}, r_{2}$ are shown in Fig. $1, \bar{R}$ is an

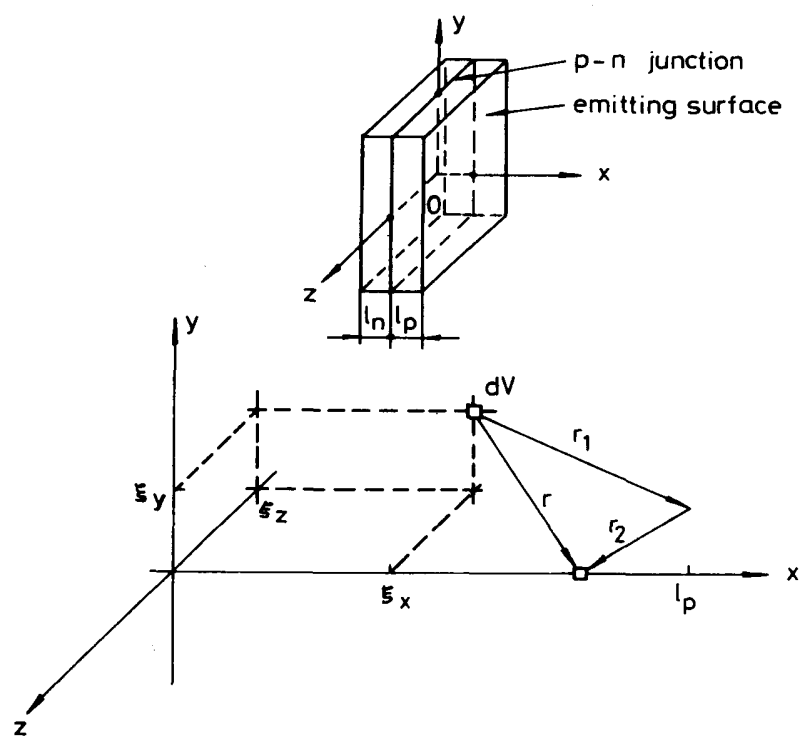

Fig. 1 Schematic diagram of p-n junction LED

$\xi_{x}, \xi_{y}$ and $\xi_{z}$ are auxiliary co-ordinates of the volume element $d V$, and $x, y$ and $z$ are basic co-ordinates of the system. $r, r_{1}$ and $r_{2}$ denote lengths of paths connecting the volume element $d V$ with the point $(x, 0,0)$ directly, and after, the reflection from the interface average reflectivity of the semiconductor-air interface. The term $\operatorname{div}_{x, y, z} G_{i, r}$ denotes that derivations in operator $\operatorname{div} \boldsymbol{G}_{\boldsymbol{i}, \boldsymbol{r}}$ are performed on co-ordinates $x, y, z$.

Evaluation of the photon absorption rate according to eqns. 9 and 10 , using eqns. 11 and 12 , allows determination of the carrier excitation rate, and solution of the eqn. 4 . The photon generation rate $g^{(2)}(x, \epsilon)$ is determined following this procedure.

\section{Simplified calculation of photon generation rate}

The basic problem in the calculation outlined above is the determination of the photon absorption rate according to eqn. 10. The evaluation of the integral in this expression can only be made by a numerical method, and consequently the obtained solution is not of an analytical form suitable for the solution of eqn. 4. The exact definition of the photon absorption rate component $v_{r}(x, \epsilon)$ is the second problem in the procedure. Eqn. 12 does not include multiple internal reflections of radiation, which form, in many cases, the dominant contribution to the photon generation rate.

When the multiple internal reflections are negligible, the calculation can be simplified by the following consideration. Supposing that the photon generation rate $g^{(2)}(x, \epsilon)$ is a series

$$
\begin{aligned}
g^{(2)}(x, \epsilon)= & g^{(1)}(x, \epsilon)+a_{0}(\epsilon)+a_{1}(\epsilon) x \\
& +a_{2}(\epsilon) x^{2} \ldots
\end{aligned}
$$

This function may be expressed in the form

$$
g^{(2)}(x, \epsilon)=g^{(1)}(x, \epsilon)+\eta r_{e}(0)+\eta\left\{r_{e}\left(l_{p}\right)-r_{e}(0)\right\} \frac{x}{l_{p}}
$$

where only the first-order term is considered. The evaluation of the integral of eqn. 10 for the values $v(0, \epsilon)$ and $v\left(l_{p}, \epsilon\right)$, determining values $r_{e}(0)$ and $r_{e}\left(l_{p}\right)$, respectively, can be made more easily than for the value $v(x, \epsilon)$.

The functions $g^{(1)}(x, \epsilon) / A$ and $g^{(2)}(x, \epsilon) / A$ are shown in Fig. 2 curves $a$ and $b$, respectively. Both functions are computed for $\epsilon=1.446 \mathrm{eV}, l_{p}=10 \mu \mathrm{m}, \alpha_{p}(\epsilon)=8430 \mathrm{~cm}^{-1}$, $\cdot \bar{R}=0.88, L_{n}=3 \mu \mathrm{m}, f(\epsilon)=7.75, h(\epsilon)=0.843, \eta=0.87$.

The contribution of the re-excitation to the photon generation rate calculated from the approximate relation, eqn. 14 , has, however, a negligible influence on the increase in the emitted photon flux, because multiple internal reflections of light in the diode volume have been neglected. Eqn. 14 originates from the exact definitions in eqns. 10 and 12 . More realistic results involving multiple internal reflections may be obtained using the following simple consideration.

The absorption of light after multiple internal reflections provides a dominant contribution to the photon absorption rate. It can be assumed that multiple internally reflected photons uniformly fill an absorption region of the diode, and consequently the photon absorption rate is constant inside this region. The $p$-region of the diode is considered to be the absorption region because the $n$-region has an absorption coefficient approximately one order of the magnitude lower than the $p$-region. Only a small part of the radiation is emitted from the GaAs surface-emitting LED. This is given by a small angle of the total reflection, and its value is about $1.3 \%$ of the total photon flux reaching any surface of the diode. Thus, it can be assumed that nearly all radiation generated in the diode is absorbed in the absorption region. The photon absorption rate then has the form

$$
v(\epsilon)=\frac{1}{l_{p}} \int_{0}^{l_{p}} g^{(1)}(x, \epsilon) d x=\frac{A L_{n}}{l_{p}} f(\epsilon)\left(1-e^{-l_{p} / L_{n}}\right)
$$


The function $g^{(2)}(x, \epsilon)$ may now be written in the form

$$
g^{(2)}(x, \epsilon)=g^{(1)}(x, \epsilon)+\eta A H \frac{L_{n}}{l_{p}}\left(1-e^{-l_{p} / L_{n}}\right)
$$

where

$$
H=\int_{0}^{\infty} h(\epsilon) f(\epsilon) d \epsilon
$$

The function $g^{(2)}(x, \epsilon)$, given by eqn. 16 , multiplied by $A^{-1}$ is plotted on Fig. 2 (curve $c$ ) using the same parameters as in the former cases.

\section{Influence of re-excitation on LED external quantum efficiency}

The re-excitation effect has been discussed in the preceding Section. Secondary excited electrons radiatively recombine and generated photons increase the photon flux emitted from the diode. As a result, the LED external quantum efficiency increases, and so this phenomenon must be considered in the calculation of the external quantum efficiency.

The external quantum efficiency can be evaluated using the known photon generation rate. On the other hand, the internal quantum efficiency can be calculated using the measured external efficiency.

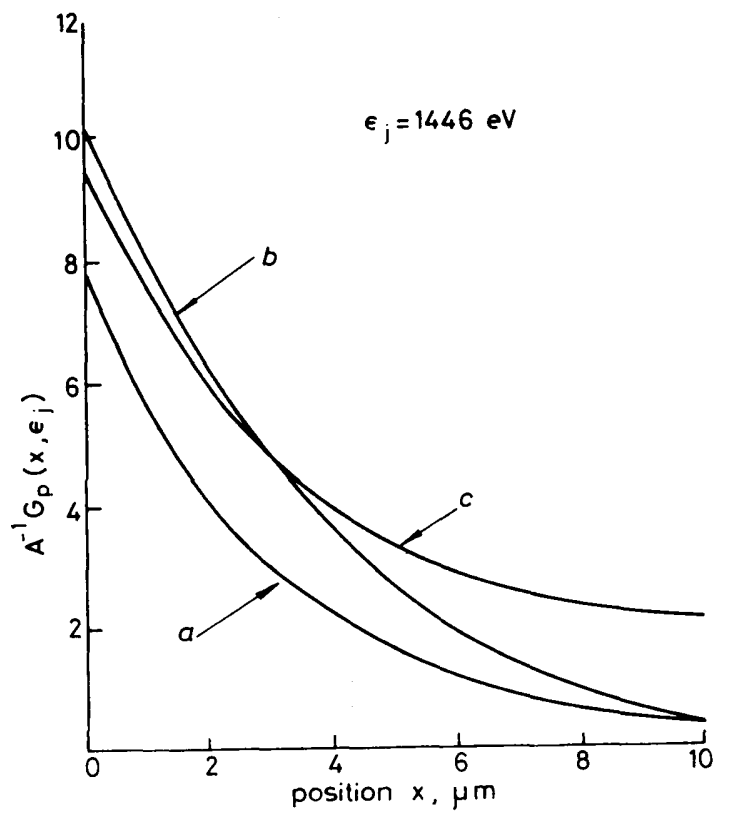

Fig. 2 Photon generation rate as function of co-ordinate $x$ $a$ function $g^{(1)}(x, \epsilon) ; b$ function $g^{(2)}(x, \epsilon)$ calculated according to eqn. 14; $c$ function $g^{(2)}(x, \epsilon)$ calculated under assumption of constant absorption rate according to eqn. 16 . The parameters of these curves are given in the text.

The basic step of the calculation is the determination of the photon flux emitted from LED. This flux may be written for the used model of LED in the form [8]

$$
\Phi=\frac{S \beta T \gamma}{4 n^{2}} \int_{0}^{\infty} \int_{0}^{l_{p}} g^{(2)}(x, \epsilon) \exp \left\{-\alpha_{p}(\epsilon)\left(l_{p}-x\right)\right\} d x d \epsilon
$$

where $S$ is the $p$ - $n$ junction area, $T$ the transmissivity of the semiconductor-air interface, $n$ a refractive index of the semiconductor, $\beta$ represents losses due to contacts on the emitting surface, $\gamma$ is a coefficient taking into consideration a nonperfection of the emitting surface which decreases reflection losses $(\gamma>1)$.
The DC external quantum efficiency is defined as

$$
\eta_{\text {ext }}=\frac{\Phi}{\Phi_{i d}}=\frac{e \Phi}{I}
$$

where $\Phi_{i d}$ is the photon flux emitted by a diode having an external quantum efficiency equal to one. Substituting eqn. 16 in eqns. 18 and 19 , one may obtain

$$
\begin{aligned}
\eta_{\text {ext }}= & \frac{\eta \beta T \gamma}{4 n^{2}} \frac{1}{1+\frac{\tau_{n} N_{A} L_{p}}{\tau_{p} N_{D} L_{n}}} \\
& \times \int_{0}^{\infty}\left[f(\epsilon) \frac{\exp \left\{-l_{p} / L_{n}\right\} \exp \left\{-\alpha_{p}(\epsilon) l_{p}\right\}}{\alpha_{p}(\epsilon) L_{n}-1}\right. \\
& \left.+\eta H\left\{1-\exp \left(-l_{p} / L_{n}\right)\right\} \frac{1-\exp \left\{-\alpha_{p}(\epsilon) l_{p}\right\}}{\alpha_{p}(\epsilon) l_{p}}\right] d \epsilon
\end{aligned}
$$

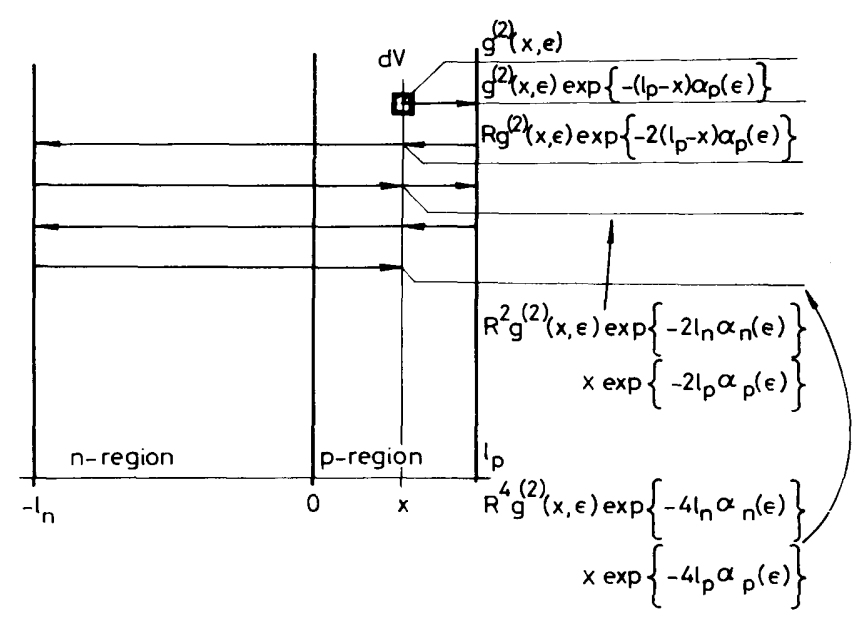

Fig. 3 Schematic diagram of multiple internal reflections of light inside diode volume

The integral in eqn. 20 must be solved numerically using an experimentally obtained absorption coefficient $\alpha_{p}(\epsilon)$ [9].

The multiple internal reflections between the front and back surfaces of the diode play an important role in the process of light emission from the diode. The emitted photon flux is assumed to originate from perpendicularly travelling photons only (Fig. 3). The reason for this assumption is a small critical angle of the internal reflection (in the case of GaAs $\Theta_{c}=16^{\circ}$ ). Then the light paths in the diode volume can be considered to equal the widths of the $p$-and $n$-regions of the diode $l_{p}$ and $l_{n}$, respectively. Supposing that the front and back diode surfaces have the same reflectivity, particular contributions to the generation photon rate caused by reflected light give the geometrical set having the sum

$$
\begin{aligned}
& g_{r}^{(2)}(x, \epsilon)=g^{(2)}(x, \epsilon) \\
& \quad \times \frac{1+R \exp \left\{-2 \alpha_{n}(\epsilon) l_{n}\right\} \exp \left\{-2 \alpha_{p}(\epsilon) x\right\}}{1-R^{2} \exp \left[-2\left\{\alpha_{n}(\epsilon) l_{n}+\alpha_{p}(\epsilon) l_{p}\right\}\right]}
\end{aligned}
$$

where $\alpha_{n}$ is the absorption coefficient of the $n$-region, and $R$ is the reflectivity of the semiconductor-air interface for the perpendicular incidence.

Substituting eqn. 21 in eqn. 18, the external quantum efficiency, including the multiple internal reflections of 
radiation, may be obtained in the form

$$
\begin{aligned}
\eta_{\text {ext }}= & \frac{\eta \beta T \gamma}{4 n^{2}} \frac{1}{1+\frac{\tau_{n} N_{A} L_{p}}{\tau_{p} N_{D} L_{n}}} \int_{0}^{\infty} \frac{1}{1-R^{2} \exp \left[-2\left\{\alpha_{n}(\epsilon) l_{n}+\alpha_{p}(\epsilon) l_{p}\right\}\right]} \\
& \times\left\{f(\epsilon) \frac{\exp \left(-l_{p} / L_{n}\right)-\exp \left\{-\alpha_{p}(\epsilon) l_{p}\right\}}{\alpha_{p}(\epsilon) L_{n}-1}+f(\epsilon) \frac{R \exp \left\{-2 \alpha_{n}(\epsilon) l_{p}\right\}\left(1-\exp \left[-\left\{\alpha_{p}(\epsilon)+1 / L_{n}\right\}\right] l_{p}\right)}{\alpha_{p}(\epsilon) L_{n}+1}\right. \\
& \left.+H \eta \frac{1-\exp \left(-l_{p} / L_{n}\right)}{\alpha_{p}(\epsilon) l_{p}}\left[1-\exp \left\{-\alpha_{p}(\epsilon) l_{p}\right\}\right]\left[1+R \exp \left\{-2 \alpha_{n}(\epsilon) l_{n}\right\} \exp \left\{-\alpha_{p}(\epsilon) l_{p}\right\}\right]\right\} d \epsilon
\end{aligned}
$$

\section{Numerical results and discusssion}

The theoretically derived relations were numerically evaluated for the case of a $\mathrm{GaAs}(\mathrm{Zn}, \mathrm{Te})$ surface-emitting LED with the following parameters: donor and acceptor concentrations $N_{D}=3 \times 10^{23} \mathrm{~m}^{-3}, N_{A}=1.6 \times 10^{24} \mathrm{~m}^{-3}$; electron and hole lifetimes were determined from References 10 and 11 as $\tau_{n}=10^{-8} \mathrm{~s}, \tau_{p}=10^{-7} \mathrm{~s}$, respectively; diffusion lengths were estimated from Reference 12 as $L_{n}=3.16 \mu \mathrm{m}$ and $L_{p}=8 \mu \mathrm{m}$; absorption coefficients $\alpha_{n}(\epsilon)$ and $\alpha_{p}(\epsilon)$ were taken from Reference 9 and a photon generation spectrum $f(\epsilon)$ from Reference 13. Computed parameters: $l_{p}=10 \mu \mathrm{m}, l_{n}=$ $100 \mu \mathrm{m}, \beta=0.7, \gamma=1, T=0.66, n=3.6, \eta=0.87, R=$ 0.34 . The constant $k$ in the expression for the quantum efficiency of the internal photoelectric phenomenon $h(\epsilon)$ was estimated to be $k=10^{-6} \mathrm{~m}$. Using the spectrum $f(\epsilon)$ from Reference 13 and the absorption coefficient from Reference 9, the value $H$ has been obtained from eqn. 17 , and is equal to 0.47 .

Values of the external quantum efficiency were evaluated for four cases:

(i) without either re-excitation or multiple reflections

(ii) without re-excitation but with multiple reflections

(iii) with re-excitation but without multiple reflections

(iv) with both re-excitation and multiple reflections. Numerical results for these four cases are given in Table 1 .

Table 1: Values of external quantum efficiency

\begin{tabular}{lll}
\hline$\eta_{\text {ext }}$ & \multicolumn{2}{l}{ Computation conditions } \\
\cline { 2 - 3 } & reexcitation & multiple reflections \\
\hline$\%$ & & - \\
0.075 & - & + \\
0.087 & - & - \\
0.122 & + & + \\
0.137 & + & \\
\hline
\end{tabular}

The first row in Table 1 was obtained by the evaluation of the relation [8]

$$
\begin{aligned}
\eta_{\text {ext }}= & \frac{\eta \beta T \gamma}{4 n^{2}} \frac{1}{1+\frac{\tau_{n} N_{A} L_{p}}{\tau_{p} N_{D} L_{n}}} \\
& \int_{0}^{\infty} f(\epsilon) \frac{\exp \left(-l_{p} / L_{n}\right)-\exp \left\{-\alpha_{p}(\epsilon) l_{p}\right\}}{L_{n} \alpha_{p}(\epsilon)-1} d \epsilon
\end{aligned}
$$

representing the external efficiency of a diode computed with neither re-excitation nor multiple reflections. This relation can be obtained substituting eqns. 1 and 2 in eqns. 18 and 19 . The shortened eqn. 22 , with only the first two terms in the sum, has been used for evaluation of the second row in Table 1. The third row in the Table was evaluated according to eqn. 20 , and the fourth according to eqn. 22.

An analysis of LED properties, mainly the external quantum efficiency, is a very complicated problem: there are many effects influencing its value. It may be concluded, on the basis of our analysis, that re-excitation has a significant contribution to the value of the external quantum efficiency. It has been calculated that this contribution is about $60 \%$ (see Table 1). Multiple reflections provide another significant contribution to the external quantum efficiency. The photon flux reaching the semiconductor-air surface is partially reflected, and, after a reflection from the back surface, increases the emitted photon flux. This contribution is, according to Table 1 , about $12 \%$.

In practical cases the reflectivity of the back surface (interface $n$-region - contact layer) is rather low, owing to the thermal treatment of the contact layer [14]. Thus multiple reflections do not occur in such diodes. The reflectivity of the back surface can be increased by application of a dielectric layer between the semiconductor and metallic layers. The ratio of the block area (necessary to ensure a reliable electrical contact and heat sink) to the whole junction area is equal $1: 10$. In this case, the experimentally measured increase in the external quantum efficiency is about 50\% [14]. This value is higher than that theoretically evaluated by us, but the reflectivity of back surface with a dielectric film is higher than the reflectivity of the semiconductor-air interface. Our calculations consider the same reflectivity on both interfaces.

Values of the external quantum efficiency, excluding re-excitation, are unrealistically small (Table 1). But those including re-excitation $(0.122 \%$ and $0.137 \%$ without and with multiple reflections, respectively) are comparable with experimentally measured values in the case of a diffused GaAs surface-emitting LED.

\section{Conclusions}

The theory of photon-feedback carrier excitation has been presented in this paper. The theoretical base describing the nature of re-excitation is given in Section 2. The simplified method for calculation, assuming a homogeneous absorption of photons inside a diode, has been used for practical purposes. Under this simplification a practical formula for the LED external quantum efficiency has been derived. Attention has been paid to multiple reflections of light inside the volume of a diode, because this phenomenon supports the assumption of the homogeneous photon absorption.

Our calculations have shown that re-excitation contributes significantly to the value of the external quantum efficiency of incoherent electroluminescent diodes. Obtained analytical results may be used for the study of superluminescent LEDs.

\section{$7 \quad$ References}

1 CARR, W.N.: 'Photometric figures of merit for semiconductor luminescent sources operating in spontaneous mode', Infrared Phys., 1966,4 , pp. $1-36$

2 KAMEDA, S., and CARR, W.N.: 'Self-excited luminescence in GaAs', J. Appl. Phys., 1973, 44, pp. 2910-2914

3 STERN, F., and WOODALL, J.H.: 'Photon recycling in semiconductor lasers', ibid., 1974, 45, pp. $3904-3906$ 
4 KURIYAMA, T., KAMIYA, T., and YANAI, H.: 'Effect of photon recycling on diffusion length and internal quantum efficiency in $\mathrm{Al}_{x} \mathrm{Ga}_{1} x_{x} \mathrm{As}-\mathrm{GaAs}$ heterostructures', Jpn.J.Appl.Phys., 1977, 16, pp. $465-477$

5 EPIFANOV, M.S., GALKIN, G.N., BOBROVA, E.A., and VAVILOV, V.S.: 'Photon transition of non-equilibrium charge carriers excitation in GaAs', Phys.Techn.Semiconductors, 1977, 11 pp. $75-80$

6 KUČERA, L., MIŠK, J., and MACHÁČ, J.: 'Contribution to the problem of the LED generation spectrum determination'. Proceedings of RECON '79 Conference, Prague, Czechoslovakia, 1979 , pp. $123-128$

7 ALFEROV, Z.I., AGAFONOV, V.G., GARBUZOV, D.Z., DAVIDUK, N.YU., and HALFIN, V.B.: 'Multiway heterostructures: II. External quantum efficiency of emission', Phys. Techn. Semiconductors, 1976, 10, pp. 1497-1506

8 KUČERA, L.: 'Frequency response of spontaneous mode electroluminescent diode at low injection levels', IEEE Trans., 1969, ED-16, pp. 303-309

9 CASEY, H.C., JR., SELL, D.D., and WECHT, K.W.: 'Concentration dependence of the absorption coefficient for $n$ - and $p$-type GaAs between 1.3 and $1.6 \mathrm{eV}$, J. Appl. Phys., 1975, 46, pp. 250-257

10 ACKET, G.A., NIJMAN, W., and LAM, H.T.: 'Electron lifetime and diffusion constant in germanium-doped gallium arsenide', $J$. Appl. Phys., 1974, 45, pp. 3033-3040

11 HWANG, C.J.: 'Quantum efficiency and radiative lifetime of the band-to-band recombination in heavily-doped $n$-type GaAs', Phys. Rev., 1972, B6, pp. 1355-1359

12 CASEY, H.C. JR., MILLER, B.I., and PINKA, E.: 'Variation of minority-carrier diffusion length with carrier concentration in GaAs liquid-phase epitaxial layers', J. Appl. Phys., 1973, 44, pp. 1281 1287

13 CARR, W.N., and BIARD, J.R.: 'Optimal generation spectrum for the electron thermal-injection mechanism in GaAs diodes', ibid., 1964,35 , pp. $2777-2779$

14 FREMUNT, R., KRATĚNA, L., MALINA, V., and NOHAVICA, D.: 'Application of $\mathrm{SiO}_{2}$ reflection layer in GaAs:Si LEDs'. Proceedings of RECON '79 conference, Prague, Czechoslovakia, 1979, pp. $62-68$

\section{Appendix}

The partial photon absorption rate may be defined as a negative divergence of the partial photon flux contributed by the volume element $d V$

$$
d v(x, \epsilon)=-\operatorname{div} G_{\substack{y=0 \\ z=0}} d V
$$

where the photon flux $G$ is given by expr. 11 and/or eqn. 12 . The operator div is evaluated with co-ordinates $x, y, z$ at the point $(x, 0,0)$. To obtain the resulting photon absorption rate it is necessary to integrate the eqn. 24 over the whole diode generation region with the auxiliary co-ordinates $\xi_{x}, \xi_{y}, \xi_{z}$, whereas the point $(x, 0,0)$ remains fixed. This operation gives eqn. 10 . 